\title{
Speculations, knowledge, and evidence about crown and root fractures
}

Eyal Rosen ${ }^{1 *}$, Igor Tsesis ${ }^{1}$ and Aviad Tamse ${ }^{2}$

Crown and root originating fractures are the main fractures occurring in teeth. Crown origination fracture (COF) usually occur in premolars and molars, in nonendodontically treated teeth (vital and non-vital) with symptoms related to the diseased pulp, while rootoriginating fractures (ROF) occur in root canal-treated teeth with or without coronal restoration and symptoms related to chronic or acute periapical disease (Bakland \& Tamse 2015).

Although in recent years, more information regarding these two entities was published in the scientific journals; there is still confusion and perplexity in the literature regarding the etiology, pathogenesis, and clinical manifestations of these entities (Bakland and Tamse 2015).

COFs include craze lines, fractured cusp, cracked tooth, and split tooth. Craze lines that are restricted to the enamel are usually not associated with pulpal or periapical pathology and may have only mild aesthetic concerns. Fractured cusp, cracked tooth, and split tooth describe longitudinal or variations thereof of COFs (Bakland and Tamse 2015; Kang et al. 2016).

The diagnosis of COFs is not straight forward because the symptoms are diverse, and it may be difficult to clinically detect the fracture lines. The detection of the fracture requires strict visual examination using magnification and illumination and sometimes even removal of coronal restorations for observation and staining using appropriate staining materials such as methylene blue dye (Kang et al. 2016).

Occlusal loads were suggested as a possible etiology for COFs; however, the exact relationship between occlusal loads and COFs development is not fully elucidated (Kim et al. 2013; Lynch and McConnell 2002).

It had been reported that patient elder age may be associated with increased risk of COFs, where most COFs are diagnosed in patients aged $50-60$ years or over 60 (Kang et al. 2016; Cameron 1976).

\footnotetext{
* Correspondence: dr.eyalrosen@gmail.com

${ }^{1}$ Department of Endodontology, The Maurice and Gabriela Goldschleger

School of Dental Medicine, Tel Aviv University, Tel Aviv, Israel

Full list of author information is available at the end of the article
}

Root-originating fracture (ROF) is a dental complication initiated from various procedures performed in the root canal space. They are manifested clinically years after endodontic treatment, are difficult to prevent, and result in hopeless prognosis for the tooth or the involved root (Tamse et al. 2015).

In 2008, the AAE had stated (AAE Colleagues for Excellence 2008) that the combination of a sinus tract and an isolated probing defect in an endodontically treated tooth with or without a dowel is pathognomonic for a ROF. Unfortunately, this "pathognomonic combination" does not occur often because ROFs mimic other clinical endodontic and periodontal entities with a variety of clinical manifestations. As a result, accurate and timely diagnosis of a ROF, which is crucial to prevent bone loss due to the fracture, is still confusing and illusive at times for the clinician (Tamse et al. 2015; Tsesis et al. 2010).

In addition, a systematic review (Tsesis et al. 2010) showed the lack of evidence-based data to support the usefulness of the current clinical and radiographic evaluation methods for the diagnosis of ROFs. However, there are several clinical studies published in recent years, even though not of the very high level of evidence, to support the AAE's 2008 statement regarding the alleged pathognomonic combination of a sinus tract and a deep probing defect for the diagnosis of ROFs.

New diagnostic modalities such as cone beam computed tomography (CBCT) were proposed for the diagnosis of ROF. However, the whole issue of the usefulness and efficacy of the CBCT for endodontic diagnosis is yet not fully understood (Rosen et al. 2015). In fact, recent meta-analysis studies and systematic reviews (Chang et al. 2016; Corbella et al. 2014), regarding the usefulness of $\mathrm{CBCT}$ scans to diagnose correctly ROFs, did not show that CBCT scans were reliable to accurately diagnose ROFs.

The etiology of ROFs in endodontically treated teeth is complex due to its multifactorial nature (Tamse et al. 2015). There are predisposing factors to ROFs and contributing ones. Some of the predisposing factors are 
the specific anatomy of the susceptible roots, the biochemical changes in the root dentin in the endodontically treated tooth, and the loss of healthy tooth substance as a result of caries and trauma before the endodontic procedures had been initiated (Tamse et al. 2015).

The contributing factors to ROFs are attributed to the iatrogenic complications that are associated with the various dental procedures, such as the removal of large amounts of sound dentin during the endodontic and restorative procedures in the root canal. The reduction of tooth stiffness as a result of the endodontic procedures and the stress generated by lateral condensation of gutta-percha followed by dowel selection and placement are examples for stress-generating procedures in the root canal (Tamse et al. 2015).

However, the majority of research done on these issues has been performed on extracted teeth and these studies are not necessarily comparable to the situation in vivo.

Restorative procedures, following root canal therapy such as dowel space preparation, their selection and the traumatic sitting of the dowels especially in the susceptible teeth and roots are additional iatrogenic-contributing etiologies for ROFs in the endodontically treated teeth (Tamse et al. 2015).

The use of fiber posts in the endodontically treated tooth is increasing in recent years due to the fact that their modulus of elasticity is similar to dentin, which allows them to flex with the root when under stress. Cagidiaco et al. (Cagidiaco et al. 2008) showed that the placement of the fiber post did improve the survival rate of endodontically treated premolars. Other studies showed the preference of fiber posts with different coronal coverage. Ferrari et al. (Ferrari et al. 2012) had pointed out the issue that the preservation of at least one coronal wall during the restoration of the endodontically treated tooth is also a significant factor to reduce fractures in maxillary premolars.

It can be concluded that to date the current availably literature regarding this significant pathological entity provides inconsistent and confusing information. In this special issue of the evidence-based endodontic journal, the most updated information about a large variety of topics related to the crown and root fractures will be covered.

Potential topics that can be included in this special issue are the following:

1) The etiology, pathogenesis, and risk factors of tooth fractures including the following:

- The role of occlusion as an etiological factor for crown and root fractures

- Dentin dehydration, macrostrains, and instrumentation effects on the initiation and propagation of fractures.
2) The clinical and radiographic presentations of teeth fractures and the use of cone beam computed tomography $(\mathrm{CBCT})$ for the diagnosis of teeth fractures.

3) Treatment alternatives for the preservation of fractured teeth.

4) The relationship between dental implants and root fractures in adjacent teeth

Authors' contributions

All authors had equal contribution to the preparation of the manuscript. All authors read and approved the final manuscript.

\section{Competing interests}

Eyal Rosen and Igor Tsesis are the editors in-chief, and Aviad Tamse is an advisory board member.

\section{Publisher's Note}

Springer Nature remains neutral with regard to jurisdictional claims in published maps and institutional affiliations.

\section{Author details}

${ }^{1}$ Department of Endodontology, The Maurice and Gabriela Goldschleger School of Dental Medicine, Tel Aviv University, Tel Aviv, Israel. ${ }^{2}$ Professor Emeritus in Endodontology Goldschlagr School of Dental Medicine, Tel-Aviv University, Tel Aviv, Israel.

Received: 12 May 2017 Accepted: 23 May 2017

Published online: 31 May 2017

\section{References}

AAE Colleagues for Excellence. Cracking the cracked tooth code: detection and treatment of various longitudinal tooth fractures. Chicago: American Association of Endodontists; 2008.

Bakland LK, Tamse A. Categorization of dental fractures. In: Tamse A, Tsesis I, Rosen $\mathrm{E}$, editors. Vertical Root Fractures in Dentistry. 1st ed. Switzerland: Springer International Publishing; 2015. p. 7-28.

Cagidiaco MC, Garcia-Godoy F, Vichi A, Grandini S, Goracci C, Ferrari M. Placement of fiber prefabricated or custom made posts affects the 3-year survival of endodontically treated premolars. Am J Dent. 2008;21(3):179-84.

Cameron CE. The cracked tooth syndrome: additional findings. J Am Dent Assoc. 1976;93(5):971-5.

Chang E, Lam E, Shah P, Azarpazhooh A. Cone-beam computed tomography for detecting vertical root fractures in endodontically treated teeth: a systematic review. J Endod. 2016:42(2):177-85.

Corbella S, Del Fabbro M, Tamse A, Rosen E, Tsesis I, Taschieri S. Cone beam computed tomography for the diagnosis of vertical root fractures: a systematic review of the literature and meta-analysis. Oral Surg Oral Med Oral Pathol Oral Radiol. 2014;118(5):593-602.

Ferrari M, Vichi A, Fadda GM, Cagidiaco MC, Tay FR, Breschi L, et al. A randomized controlled trial of endodontically treated and restored premolars. J Dent Res. 2012;91(7 Suppl):72S-8.

Kang SH, Kim BS, Kim Y. Cracked teeth: distribution, characteristics, and survival after root canal treatment. J Endod. 2016;42(4):557-62.

Kim SY, Kim SH, Cho SB, Lee GO, Yang SE. Different treatment protocols for different pulpal and periapical diagnoses of 72 cracked teeth. J Endod. 2013;39(4):449-52.

Lynch CD, McConnell RJ. The cracked tooth syndrome. J Can Dent Assoc. 2002;68(8):470-5

Rosen E, Taschieri S, Del Fabbro M, Beitlitum I, Tsesis I. The diagnostic efficacy of cone-beam computed tomography in endodontics: a systematic review and analysis by a hierarchical model of efficacy. J Endod. 2015;41(7):1008-14.

Tamse A, Tsesis I, Rosen E, editors. Vertical root fractures in dentistry. Switzerland: Springer International;2015.

Tsesis I, Rosen E, Tamse A, Taschieri S, Kfir A. Diagnosis of vertical root fractures in endodontically treated teeth based on clinical and radiographic indices: a systematic review. J Endod. 2010;36(9):1455-8. 